\title{
Highly Effective Crosstalk Mitigation Method Using Counter-Propagation in Semiconductor Optical Amplifier for Remodulation WDM-PONs
}

\author{
Quang Thai Pham \\ Department of Electrical and Electronic Engineering, Ho Chi Minh City University of Technology, Ho Chi Minh, Vietnam \\ Correspondence should be addressed to Pham Quang Thai; pqthai.hcmut@gmail.com
}

Received 23 June 2014; Revised 22 September 2014; Accepted 24 September 2014; Published 19 October 2014

Academic Editor: Luciano Mescia

Copyright (C) 2014 Pham Quang Thai. This is an open access article distributed under the Creative Commons Attribution License, which permits unrestricted use, distribution, and reproduction in any medium, provided the original work is properly cited.

\begin{abstract}
Remodulation-induced crosstalk mitigation in WDM-PON using remodulation approach is presented in this paper. Utilizing alloptical signal processing, the proposed method has been able to significantly improve system performance in terms of bit error rate (BER) and bit rate distance product. Moreover, the proposed method could be used for both baseband and modulated downstream electrical signals.
\end{abstract}

\section{Introduction}

As old copper line has been progressively replaced by optical fiber and coherent optical modulation techniques are being deployed, access networks have also changed from fiberto-the-X (FTTX) active optical network (AON) systems to passive optical network (PON) systems.

In PON, deployment and maintenance costs are greatly reduced with fewer fibers and no active components from optical line terminal (OLT) to optical network unit (ONU). With low fiber's attenuation and better sensitivity of modern optical receivers, it is possible to send optical signal across nearly $20 \mathrm{~km}$ toward up to 64 ONUs using only one single mode fiber and optical power splitter/combiner. Standardized by IEEE and ITU-T, the current PON topologies are E-PON and G-PON using time division multiplexing $[1,2]$.

However, since optical signal power is equally divided between users and time division multiplexing is used, there are issues in security, synchronization, latency, and laser's rise-time [2]. Next generation of PON focuses on wavelength division multiplexing (WDM-PON) techniques [3-7]. As each user utilizes a different wavelength, the weaknesses of traditional PON are overcome.

WDM in traditional sense, where each downstream and upstream channel occupies a different wavelength, is only suitable for backbone networks. In access networks, where user's deployment cost is limited, installing a wavelengthspecific distributed feedback (DFB) laser diode for each user is impractical. Thus, the ability to send and receive signal across a large bandwidth, or colorless operation at ONU, is highly desired [8-13]. One of the best solutions is to reuse the downstream wavelength as carrier for upstream data by means of reflective semiconductor optical amplifier (RSOA) or semiconductor optical amplifier (SOA) with electroabsorption modulator (EAM) [14, 15]. Drawbacks of this solution include remodulation-induced crosstalk at the ONU and crosstalk causes by Rayleigh backscattering along the fiber.

Since Rayleigh backscattering introduces crosstalk to counter-propagation signal of the same wavelength, it can be reduced if the upstream and downstream signal's spectra are not overlap. As a result, report on Rayleigh backscattering crosstalk mitigation utilized different line codes [16-20] or different spectral-shaping techniques [21-23] for downstream and upstream signals.

On the other hand, crosstalk at the ONU is caused by the remodulation approach itself. Since downstream optical signal is used to modulate with upstream data, residual downstream data cause remodulation-induced crosstalk. To the best of our knowledge, this type of crosstalk is usually reduced by saturating the downstream signal before remodulation [24, 25]. This method requires low extinction ratio in downstream 


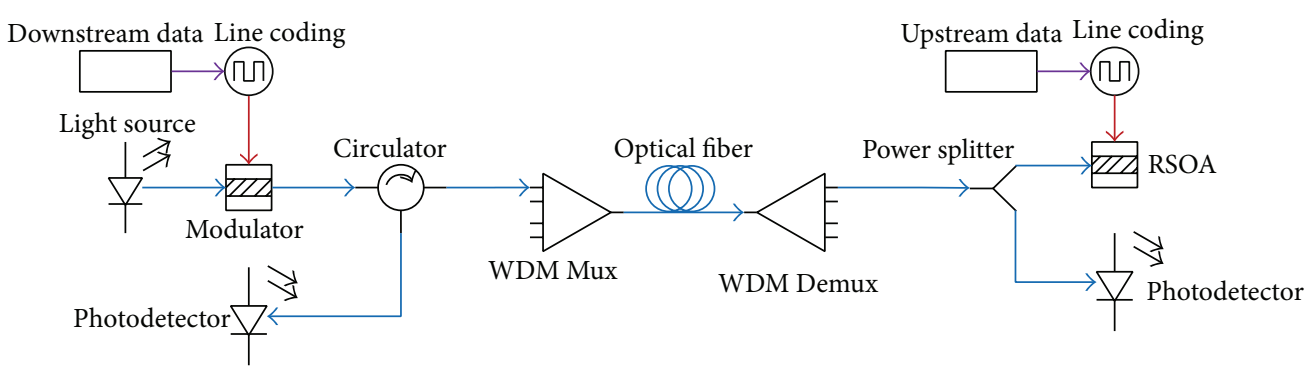

FIGURE 1: WDM-PON system with remodulation approach.

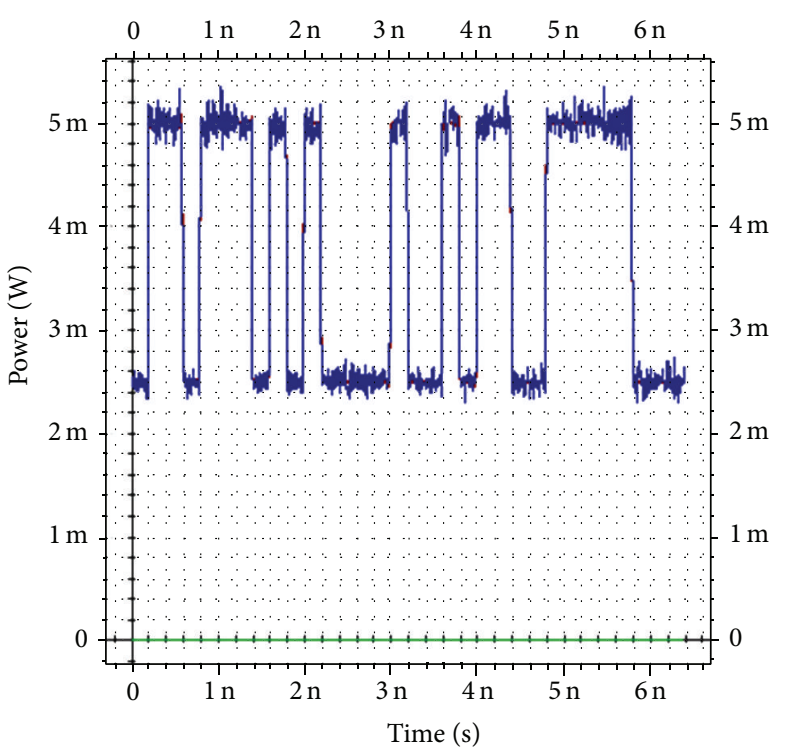

(a)

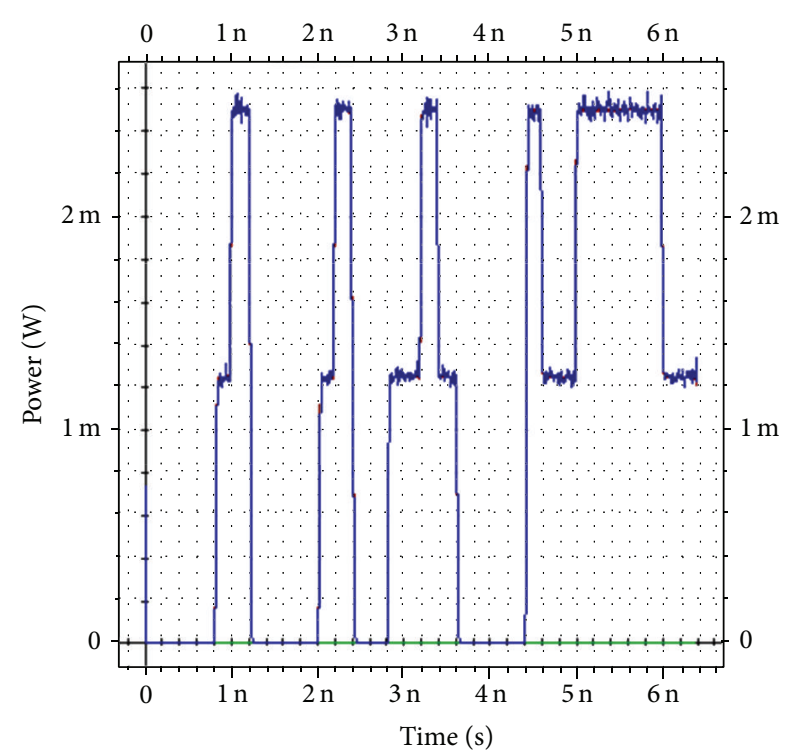

(b)

Figure 2: (a) Downstream modulated signal and (b) fluctuation caused by remodulation crosstalk in upstream modulated signal.

signal and, thus, reduces system performance in terms of BER and bit rate distance product.

In this report, we propose an all-optical approach to mitigate remodulation-induced at the ONU in remodulation approach. Utilizing cross-gain modulation in counterpropagation SOA, our proposed method was able to effectively remove residual downstream signal in upstream signal, thus reducing upstream BER. The proposed method performed well on both baseband and modulated downstream electrical signal. Moreover, factorial analysis also proved that the interaction between downstream and upstream signals on upstream BER was effectively removed for bit rate of less than 20 Gbps. Last but not least, our proposed ONU was able to achieve colorless operation.

\section{Principles}

A typical WDM-PON system applying remodulation approach is shown in Figure 1. Each pair of OLT and ONU uses the same wavelength, and there is no active device along the transmission fiber. In this figure, only one channel is depicted for better clarity. Downstream data is modulated with optical signal at OLT and sent to ONU though optical fiber. At ONU, an optical power splitter/combiner separates the received signal into two branches. The signal in one branch is used for downstream signal detection, while the other is used as optical carrier signal for upstream transmission. RSOA is usually utilized for remodulation for several reasons. First and foremost, since RSOA is an optical amplifier, it compensates some of the optical power attenuated along the transmission link. Secondly, RSOA's gain can be controlled via its applied bias current. Therefore, upstream electrical signal can be directly modulated with downstream optical signal. Thirdly, gain of RSOA typically linearly increases or decreases around $3 \mathrm{~dB}$ across C-band. Thus, downstream signals across $\mathrm{C}$-band can be remodulated and colorless operation is achieved. Finally, thanks to its structure, RSOA can easily be integrated with various silicon photonic devices such as electroabsorption modulator (EAM) to further reduce receiver's footprint and increase modulation bandwidth.

However, reusing downstream optical signal as upstream carrier signal inevitably results in crosstalk between downstream and upstream signals. Simulated upstream signal is shown in Figure 2. In this figure, NRZ line coding was used 


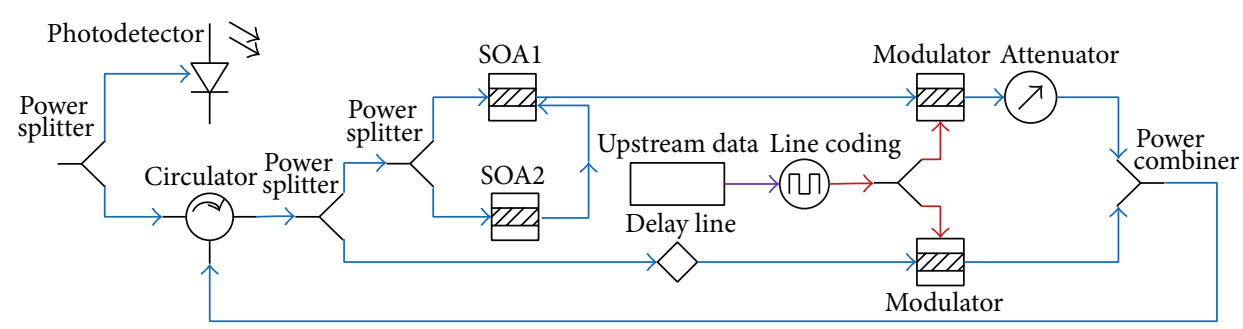

FIGURE 3: Proposed remodulation-induced crosstalk mitigation method.

for both downstream and upstream data. Upstream electrical signal was directly modulated with downstream optical signal through the RSOA. Upstream bit rate was assumed to be slower than downstream bit rate. As seen in the figure, residual downstream signal is clearly shown as fluctuations in the modulated upstream NRZ signal. Basically, this phenomenon can be compared to direct modulation of upstream signal with a very poor quality light source in terms of power fluctuation and linewidth.

Usually, the RSOA has to be operated near its saturated gain region to reduce fluctuations. However, it is impossible to completely remove the residual signal, since only the fluctuations during the high level duration of downstream bit are saturated. Thus, extinction ratio (ER) of downstream modulated signal has to be low. Additionally, since the RSOA also serves as a modulator, operation in saturated region reduces ER of upstream signal. The ER also depends on bit rate, bandwidth, and saturated gain power of the RSOA as studied in [25].

The $3 \mathrm{~dB}$ modulation bandwidth of RSOA is usually less than $2 \mathrm{GHz}$ in commercial products. This modulation bandwidth, in theories, allows up to $2.5 \mathrm{Gbps}$ NRZ signal. However, due to saturated gain operation conditions, the actual upstream bit rate in remodulation scheme using RSOA is actually smaller than its already limited modulation bandwidth. In reported literature using this approach [24], downstream signal ER was $5 \mathrm{~dB}$ for $1.25 \mathrm{Gbps}$ NRZ signal over $20 \mathrm{~km}$. In another report [25], downstream signal ER was $3 \mathrm{~dB}$ for $622.08 \mathrm{Mbps}$ NRZ signal over $40 \mathrm{~km}$. It is noted that, in comparison with ITU-T G.984.1 and G.984.2 specifications, upstream bit rate distance product in these reports is less than the currently deployed G-PON, which is 2.4 Gbps NRZ signal over $20 \mathrm{~km}$.

Our proposed method to reduce crosstalk is shown in Figure 3. In this block diagram the reused signal is further divided into two branches. In the upper branch, two SOAs, denoted as SOA1 and SOA2, are used to invert the downstream signal levels. SOA2 is operated within its linear region, while SOA1 is operated near its saturation region. Output signal after SOA2 is sent through SOA1 but in the opposite direction. By combining two counterpropagating signals with different power levels, cross-gain modulation effect in SOA1 creates an inverted version of the original signal. In the lower branch, the downstream signal is unchanged.

It is crucial for the signals in both branches to be synchronized and have the same power level, therefore, an optical attenuator is needed in the upper branch and an optical delay line is needed in the lower branch. The two signals are modulated with the same upstream electrical signal by two identical modulators, which can be SOAs or EAMs. Finally, modulated signals are combined through a coupler. The output signal is then used as upstream remodulated signal.

In principles, optical signal inversion is similar to optical logic NOT gates using SOA [26]. In such systems, two wavelengths are sent through one SOA. Cross-gain modulation effect between these two wavelengths creates an inverted version of the first wavelength in the second wavelength. This simple approach, however, changes the inverted signal's wavelength and cannot be used for colorless ONU. Therefore, counter-propagation [27] is utilized in our proposed approach to ensure no wavelength conversion. It is noted that optical NOT operation is only similar to traditional electronic NOT for OOK signals, where low level input signal becomes high level output signal and vice versa. With multilevel signal such as QAM, the optical NOT subsystem's output is the inverted version of the input signal in terms of optical power.

Using this method, fluctuations caused by downstream signal are still presented in the modulated signals in both branches. However, since the residual signals in the upper branch are inverted version of the residual signals in the lower branches, they are flattened after the coupler. Therefore, no crosstalk is introduced between the downstream and upstream signals. Although SOA1 is operated near its saturation region, the downstream optical signal only serves as pumping source to trigger cross-gain effect in SOA1. Therefore, there was no limitation in the saturated SOA as studied in [25]. As a result, upstream bit rate distance product can be improved in comparison with $[24,25]$.

Moreover, since downstream signal is removed from modulated upstream signal, the line coding formats and modulation methods for downstream and upstream electrical signals are also not limited. This is crucial to further improve system's performance. As mentioned in the first section, the most effective method to mitigate Rayleigh backscattering crosstalk at the moment is to avoid overlapping in downstream and upstream signal's spectra. Reported approaches [16-20] usually use two different line coding formats or modulation techniques. Thus, our proposed system can be combined with methods in [16-20] to mitigate both remodulation-induced crosstalk at the ONU and Rayleigh backscattering crosstalk at the same time. 
In comparison with a typical system using only one RSOA, our proposed system is more complex. However, the increase in complexity can be justified with colorless operation, better bit rate distance product for both downstream and upstream bit rates, and compatible with recent approaches in WDM-PON using complex modulated electrical signals. Moreover, since all-optical signal processing and only SOA and/or EAM are used, the proposed system can be combined into one optical integrated-circuit using current CMOS technology. Thus, cost can be reduced when the system is mass produced.

\section{Simulation Results}

The proposed crosstalk mitigation method was utilized for both NRZ and 16-QAM electrical signals to prove its capability for both baseband and modulated electrical signals. Optisystem simulation program from Optiware was used. The significance and effect of upstream bit rate, downstream bit rate, and fiber length were also analyzed using factorial analysis in Minitab statistical software.

Since there is no specification for WDM-PON at the moment, ITU-T G.984.2 specifications for current G-PON were used as references in our simulations. According to ITU$\mathrm{T}$, line coding for both downstream and upstream signals is NRZ. Maximum downstream and upstream bit rate is 2.4 Gbps. Maximum distance is $20 \mathrm{~km}$. BER of $10^{-10}$ was considered acceptable.

Therefore, for simulation with baseband electrical signal, NRZ line coding was used for both transmission directions. Downstream bit rate was increased from $2.5 \mathrm{Gbps}$ to $10 \mathrm{Gbps}$. Fiber length was increased from $10 \mathrm{~km}$ to $100 \mathrm{~km}$. Upstream signal was NRZ at $2.5 \mathrm{Gbps}$. DFB laser at $1550 \mathrm{~nm}$ and $0 \mathrm{dBm}$, which is the lower output limit of commercial G-PON OLT, was used as light source. Laser's linewidth was $10 \mathrm{MHz}$. Photo diode noise was $-68 \mathrm{dBm}$. SOA1 and SOA2 were modeled after the mathematical model in $[28,29]$ and commercial products' specifications with $20 \mathrm{~dB}$ gain, $14 \mathrm{dBm}$ saturation output power, driving current from $500 \mathrm{~mA}$ to $750 \mathrm{~mA}$, and $2 \mathrm{GHz} 3 \mathrm{~dB}$ modulation bandwidth. SOA2's driving current was $500 \mathrm{~mA}$. SOAl's driving current was $720 \mathrm{~mA}$. Upstream modulators were 2.5 Gbps EAMs.

For simulations with modulated electrical signal, the downstream bit rate was also increased from $2.5 \mathrm{Gbps}$ to 10 Gbps. The fiber length was also increased from $20 \mathrm{~km}$ to $100 \mathrm{~km}$. The upstream signal was also NRZ at $2.5 \mathrm{Gbps}$. All other devices were also the same. However, optical coherent QAM modulation and demodulation were used for downstream signal.

For comparison purpose, the system in Figure 1 was used as typical remodulation WDM-PON system using RSOA. All signals and devices had the same characteristic as in simulations with NRZ signal. The RSOA had the same characteristics as SOA1 with $99 \%$ reflectivity in one facet.

Rayleigh backscattering becomes significant for transmission length longer than $20 \mathrm{~km}$ and contributes to the overall crosstalk between downstream and upstream data. However, this study emphasizes remodulation-induced fluctuation at
ONU. Fundamentally, this is a different problem in comparison with light scattering in fibers. Therefore, in order to isolate and analyze only the crosstalk caused by remodulationinduced fluctuations, Rayleigh backscattering was ignored in our simulations unless stated otherwise. Some results with Rayleigh backscattering are only shown as references. Also, only BER of upstream signal is shown in this section since downstream signal does not suffer from remodulationinduced crosstalk.

For NRZ downstream signal, the modulated signals in two branches are shown in Figures 4(a) and 4(b), respectively. As seen in these figures, residual downstream signals are clearly shown in remodulated upstream signals. The combined signal is shown in Figure 4(c).

Similarly, for 16-QAM downstream signal, the modulated signals in two branches are shown in Figures 5(a) and 5(b), respectively. It is noted that the inverted version of received signal also had multiple levels, which was different than the concept of logic NOT in electrical domain as mentioned in the previous section. The combined signal is shown in Figure 5(c).

In these figure, downstream signal was mostly erased from remodulated upstream signal. However, even with utmost care in adjusting the delay line and attenuator, the signals in two branches could not be perfectly synchronized. Some fluctuations were still presented as seen in Figures 4(c) and 5(c). Fortunately, these fluctuations were at higher frequency in the signal's spectrum. Since the electrical bandwidth of optical receiver devices is generally considered to be low-pass Bessel filter, these fluctuations were filtered out in electrical domain.

BER of upstream NRZ signals when downstream signal was NRZ and 16-QAM is shown in Figure 6. For comparison purposes, BER of upstream NRZ signal for the RSOA system in Figure 1 was also estimated. In this figure, upstream bit rate was at $2.5 \mathrm{Gbps}$; fiber length was at $100 \mathrm{~km}$; and downstream bit rate was increased from $2.5 \mathrm{Gbps}$ to $10 \mathrm{Gbps}$.

As seen in Figure 6, upstream BER of RSOA system was around $10^{-3}$ and slightly decreased as downstream bit rate increased. This was caused by the low-pass Bessel filter response of optical receiver's electrical bandwidth. Higher frequencies of residual downstream signal were filtered out at the receiver.

Our proposed method, on the other hand, removed the downstream signal from upstream signal. Thus, acceptable BER was achieved up to 7.5 Gbps downstream NRZ signal. For faster downstream bit rate, the upstream BER quickly rose to around $10^{-6}$. This was due to the simulated SOA model's limited response time.

For 16-QAM downstream signal, our proposed method performed even better. Since the actual baud rate of 16-QAM is several times smaller than the baud rate of NRZ given the same bit rate, the SOAs can still perform adequately. Thus, BER of upstream signal was acceptable for $10 \mathrm{Gbps}$ downstream bit rate.

BER of upstream signal when downstream bit rate was at 10 Gbps is shown in Figure 7. The fiber length was increased from $30 \mathrm{~km}$ to $100 \mathrm{~km}$. Performance of the typical RSOA system was inadequate as predicted. 


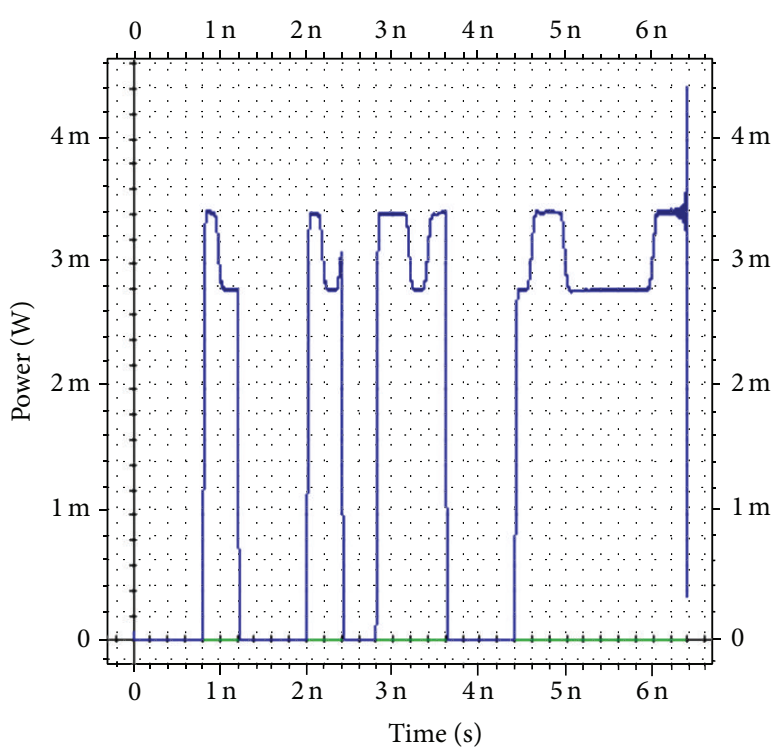

(a)

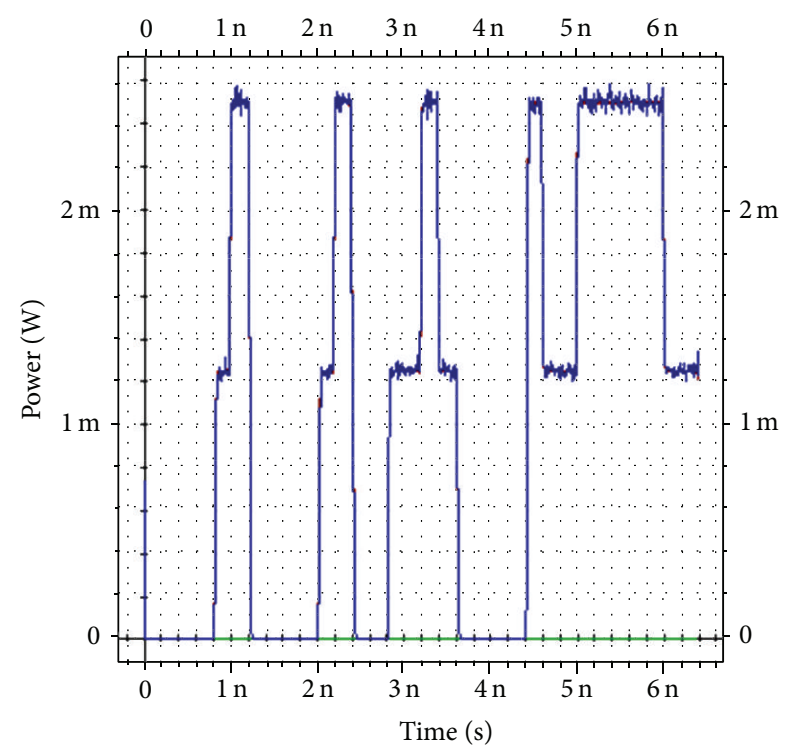

(b)

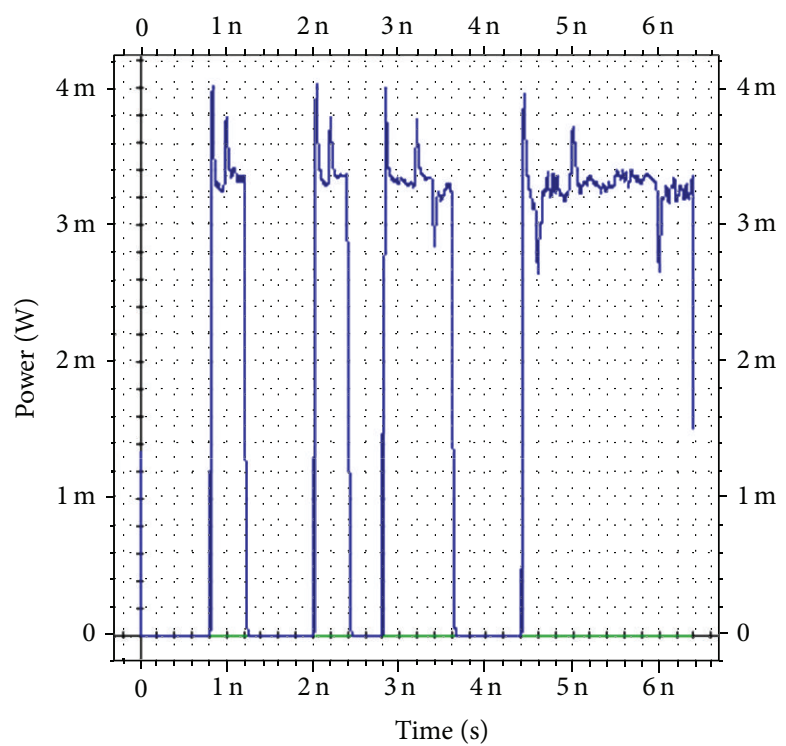

(c)

FIGURE 4: Downstream NRZ signal and (a) modulated upstream signal in upper branch; (b) modulated upstream signal in lower branch; (c) upstream signal.

The upstream BERs when downstream signal was also NRZ with and without Rayleigh backscattering are shown in this figure. With additional crosstalk from backscattering along the fiber length, acceptable length was around $30 \mathrm{~km}$. This result was better than other reported methods using RSOA saturated gain methods.

However, without Rayleigh backscattering, our proposed method could provide BER of $10^{-10}$ up to $40 \mathrm{~km}$. Therefore, it is possible to further improve performance if our proposed method is combined with other reported backscattering crosstalk mitigation methods.

Performance of upstream BER for downstream 16-QAM signal was acceptable up to $100 \mathrm{~km}$ of fiber length. As explained before, since the baud rate of 16-QAM is smaller than NRZ, the SOAs could properly invert the downstream signal and the system could remove downstream residual from upstream signal.

The significance of downstream bit rate, upstream bit rate, and fiber length on upstream BER was also analyzed using 2-level factorial analysis. The analysis space was limited within $2.5 \mathrm{Gbps}$ to $20 \mathrm{Gbps}$ for downstream signal, $2.5 \mathrm{Gbps}$ to $5 \mathrm{Gbps}$ for upstream signal, and $20 \mathrm{~km}$ to $100 \mathrm{~km}$ for fiber length. Interaction plots are shown in Figure 8. In this figure, the interactions between fiber length and downstream bit rate as well as between fiber length and upstream bit rate did not have much significance on the overall upstream BER. 


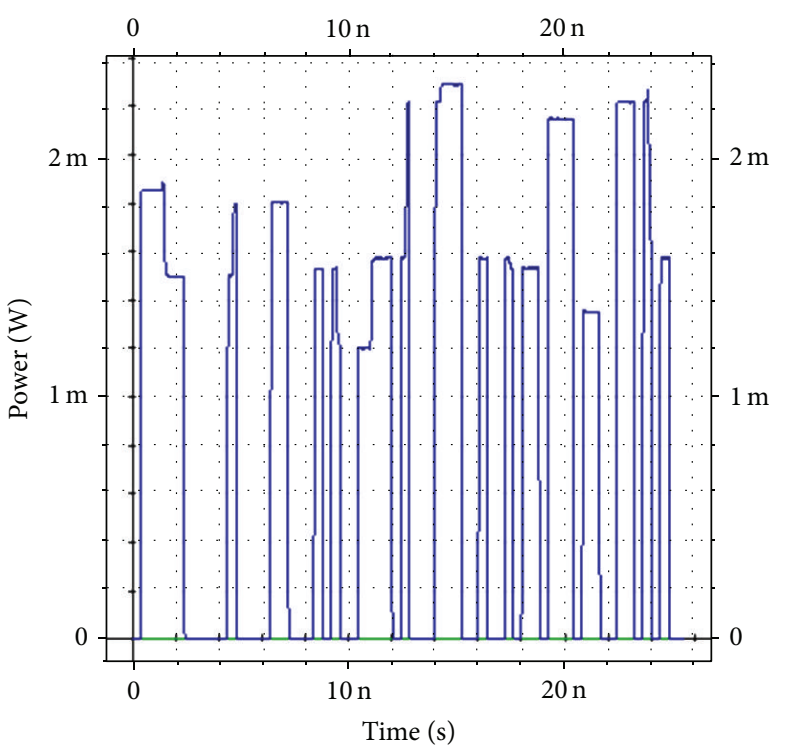

(a)

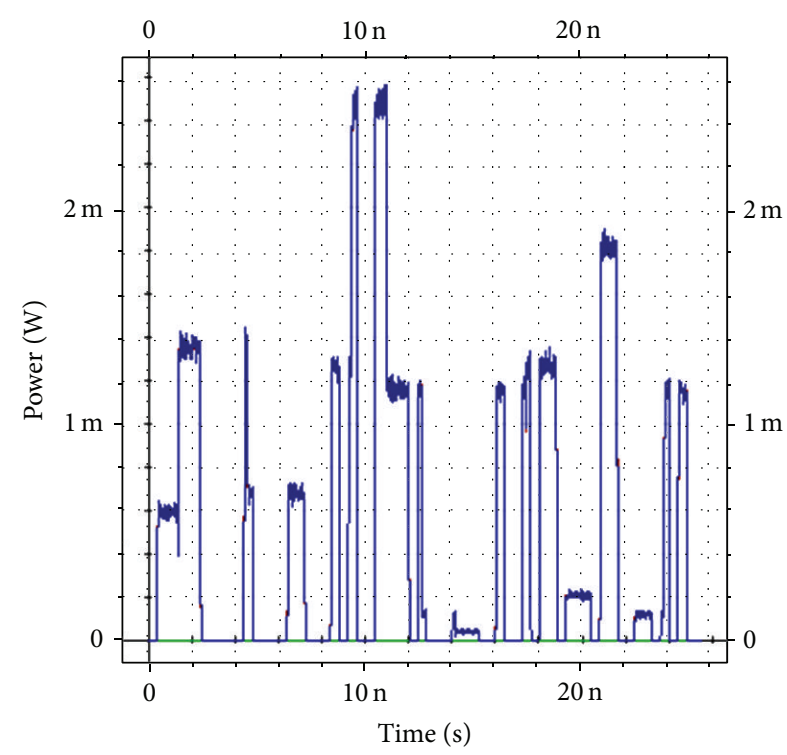

(b)

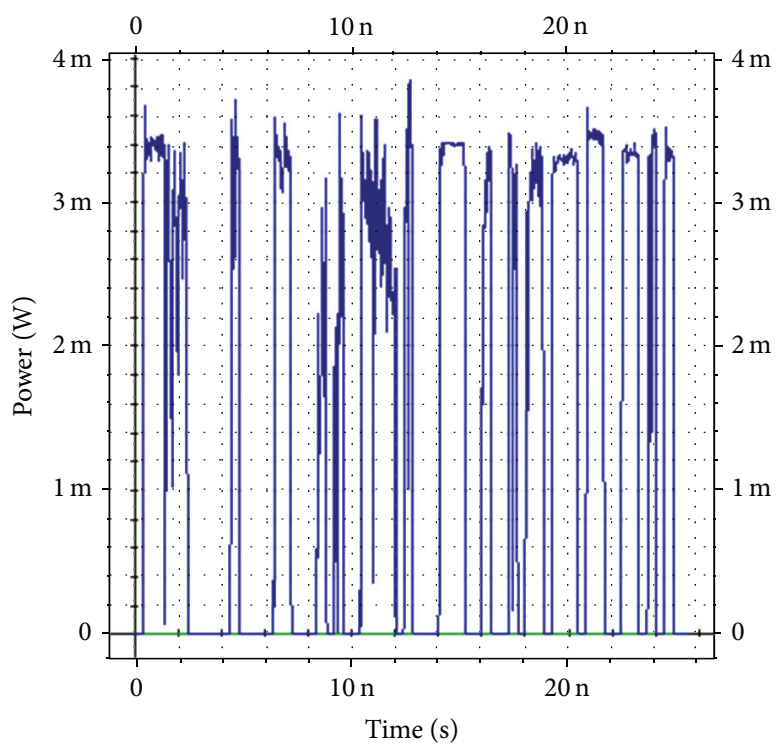

(c)

FiguRE 5: Downstream 16-QAM signal and (a) modulated upstream signal in upper branch; (b) modulated upstream signal in lower branch; (c) upstream signal.

However, as the downstream bit rate reached $20 \mathrm{Gbps}$, the interaction between downstream bit rate and upstream bit rate started to affect the upstream BER. In other word, at downstream bit rate less than $20 \mathrm{Gbps}$, our proposed method could effectively isolate the interaction between downstream and upstream signals.

\section{Conclusions}

Our proposed crosstalk mitigation method has been able to significantly improve upstream BER in WDM-PON system using remodulation approach. Using all-optical signal processing, our proposed method has performed well for both NRZ and 16-QAM electrical signals. Research on combination with reported Rayleigh backscattering crosstalk mitigation methods has been carried out to further improve the proposed system.

\section{Conflict of Interests}

The author declares that there is no conflict of interests regarding the publication of this paper. 


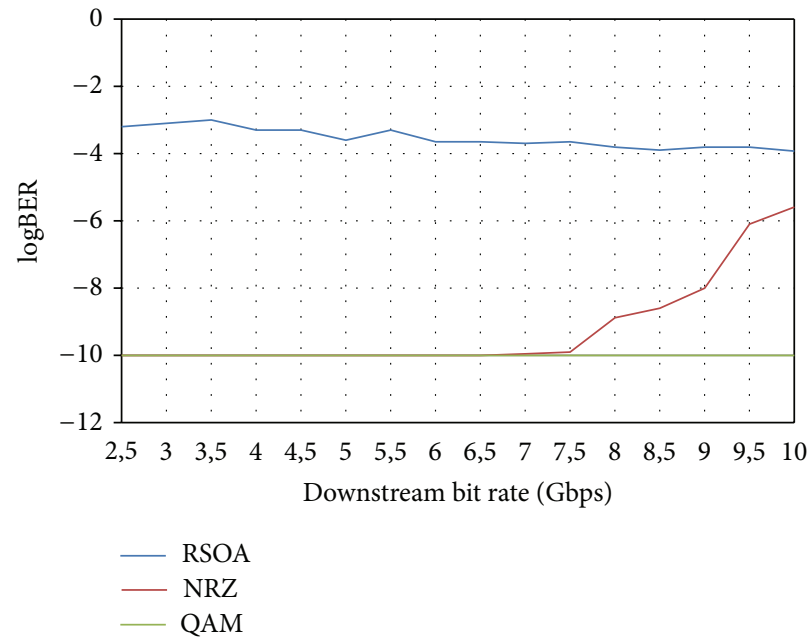

FigURE 6: Upstream logBER versus downstream bit rate when fiber length is $100 \mathrm{~km}$.

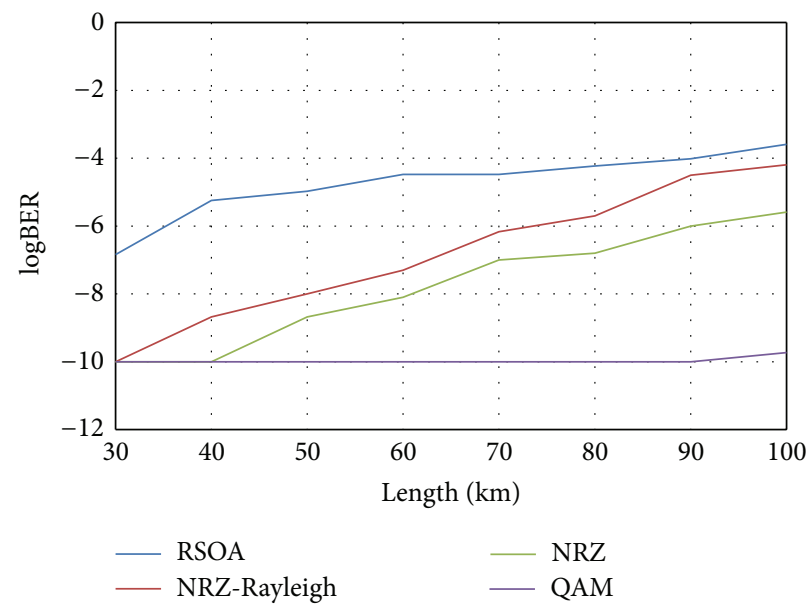

FIGURE 7: Upstream logBER versus fiber length when downstream bit rate is $10 \mathrm{Gbps}$.

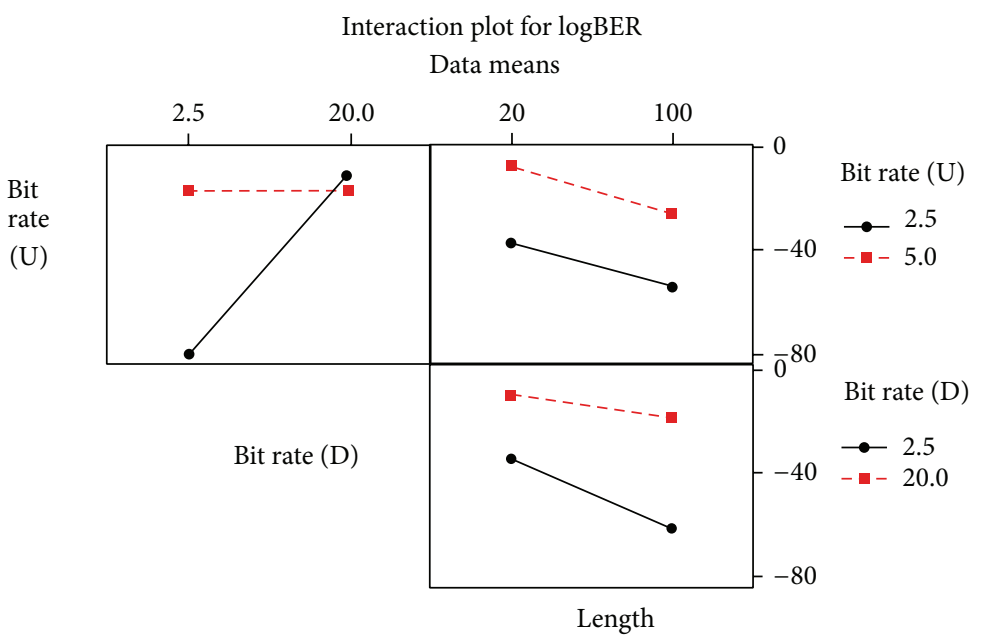

FIGURE 8: Interaction plot for $\operatorname{logBER}$ as a function of downstream bit rate, upstream bit rate, and fiber length. 


\section{Acknowledgment}

This research is funded by Vietnam National University HoChiMinh City (VNU-HCM) under Grant no. C2014-2032.

\section{References}

[1] J.-P. Elbers and K. Grobe, "Optical access solutions beyond 10GEPON/XG-PON," in Proceedings of the Conference on Optical Fiber Communication, Collocated National Fiber Optic Engineers Conference (OFC/NFOEC '10), pp. 1-3, San Diego, Calif, USA, March 2010.

[2] S. Weinstein, Y. Luo, and T. Wang, The ComSoc Guide to Passive Optical Network: Enhancing the Last Mile Access, John Wiley \& Sons, 2012.

[3] S. J. Park, C. H. Lee, K. T. Jeong, H. J. Park, J. G. Ahn, and K. H. Song, "Fiber-to-the-home services based on wavelengthdivision-multiplexing passive optical network," Journal of Lightwave Technology, vol. 22, no. 11, pp. 2582-2591, 2004.

[4] R. Gaudino, D. Cárdenas, M. Bellec et al., "Perspective in nextgeneration home networks: toward optical solutions?" IEEE Communications Magazine, vol. 48, no. 2, pp. 39-47, 2010.

[5] L. G. Kazovsky, W.-T. Shaw, D. Gutierrez, N. Cheng, and S.W. Wong, "Next-generation optical access networks," Journal of Lightwave Technology, vol. 25, no. 11, pp. 3428-3442, 2007.

[6] J. I. Kani, K. Iwatsuki, and T. Imai, "Optical multiplexing technologies for access-area applications," IEEE Journal on Selected Topics in Quantum Electronics, vol. 12, no. 4, pp. 661668, 2006.

[7] F. J. Effenberger, J.-I. Kani, and Y. Maeda, "Standardization trends and prospective views on the next generation of broadband optical access systems," IEEE Journal on Selected Areas in Communications, vol. 28, no. 6, pp. 773-780, 2010.

[8] Z. Xu, Y. J. Wen, W. D. Zhong et al., "WDM-PON architectures with a single shared interferometric filter for carrier-reuse upstream transmission," Journal of Lightwave Technology, vol. 25, no. 12, pp. 3669-3677, 2007.

[9] Z. Xu, Y. J. Wen, W.-D. Zhong et al., "High-speed WDM-PON using CW injection-locked Fabry-Pérot laser diodes," Optics Express, vol. 15, no. 6, pp. 2953-2962, 2007.

[10] N. J. Frigo, P. P. Iannone, P. D. Magill et al., "Wavelengthdivision multiplexed passive optical network with cost-shared components," IEEE Photonics Technology Letters, vol. 6, no. 11, pp. 1365-1367, 1994.

[11] D. K. Jung, S. K. Shin, C.-H. Lee, and Y. C. Chung, "Wavelengthdivision-multiplexed passive optical network based on spectrum-slicing techniques," IEEE Photonics Technology Letters, vol. 10, no. 9, pp. 1334-1336, 1998.

[12] C.-H. Lee, S.-M. Lee, K.-M. Choi et al., "WDM-PON experiences in Korea," Journal of Optical Networking, vol. 6, no. 5, pp. 451-464, 2007.

[13] K. Grobe and J.-P. Elbers, "PON in adolescence: from TDMA to WDM-PON," IEEE Communications Magazine, vol. 46, no. 1, pp. 26-34, 2008.

[14] J. H. Lee, S.-H. Cho, H.-H. Lee et al., "First commercial deployment of a colorless gigabit WDM/TDM hybrid PON system using remote protocol terminator," Journal of Lightwave Technology, vol. 28, no. 4, pp. 344-351, 2010.

[15] C. Kazmierski, "Remote amplified modulators: key components for $10 \mathrm{~Gb} / \mathrm{s}$ WDM PON," in Proceedings of the Optical Fiber
Communication (OFC), Collocated National Fiber Optic Engineers Conference (OFC/NFOEC '10), pp. 1-3, San Diego, Calif, USA, March 2010.

[16] A. Chiuchiarelli, M. Presi, R. Proietti et al., "Enhancing resilience to rayleigh crosstalk by means of line coding and electrical filtering," IEEE Photonics Technology Letters, vol. 22, no. 2 , pp. $85-87,2010$.

[17] J. J. Martínez, J. I. Garcés Gregorio, A. López Lucia, A. Villafranca Velasco, J. C. Aguado, and M. Á. Losada Binué, "Novel WDM-PON architecture based on a spectrally efficient IM-FSK scheme using DMLs and RSOAs," Journal of Lightwave Technology, vol. 26, no. 3, pp. 350-356, 2008.

[18] W. Hung, C. K. Chan, L. K. Chen, and F. Tong, "An optical network unit for WDM access networks with downstream DPSK and upstream re-modulated OOK data using injected-locked FP laser," in Proceedings of the Optical Fiber Communication Conference (OFC '03), pp. 281-282, 2003.

[19] J. M. Kang and S. K. Han, "A novel hybrid WDM/SCM-PON sharing wavelength for up- and down-link using reflective semiconductor optical amplifier," IEEE Photonics Technology Letters, vol. 18, no. 3, pp. 502-504, 2006.

[20] Z. Al-Qazwini and H. Kim, "10-Gbps single-feeder, full-duplex WDM-PON using directly modulated laser and RSOA," Proceedings of the Optical Fiber Communication Conference and Exposition and the National Fiber Optic Engineers Conference (OFC/NFOEC '12), Paper OTh1F.5 pages, 2012.

[21] C. Kottke, J. Von Hoyningen-Huene, M. Eiselt et al., "Coherent SCM-WDM-PON system using OFDM or single carrier with SSB modulation and wavelength reuse," in Proceedings of the 39th European Conference and Exhibition on Optical Communication (ECOC '13), pp. 570-572, September 2013.

[22] D. Lavery, M. Paskov, and S. J. Savory, "Spectral shaping for mitigating backreflections in a bidirectional $10 \mathrm{Gbit} / \mathrm{s}$ coherent WDM-PON," in Proceedings of the Optical Fiber Communication Conference and Exposition and the National Fiber Optic Engineers Conference (OFC/NFOEC '13), pp. 1-3, March 2013.

[23] Z. Al-Qazwini and H. Kim, "Symmetric 10-Gb/s WDM-PON using directly modulated lasers for downlink and RSOAs for uplink," Journal of Lightwave Technology, vol. 30, no. 12, Article ID 6175913, pp. 1891-1899, 2012.

[24] W. Lee, M. Y. Park, S. H. Cho et al., "Bidirectional WDMPON based on gain-saturated reflective semiconductor optical amplifiers," IEEE Photonics Technology Letters, vol. 17, no. 11, pp. 2460-2462, 2005.

[25] H. Takesue and T. Sugie, "Wavelength channel data rewrite using saturated SOA modulator for WDM networks with centralized light sources," Journal of Lightwave Technology, vol. 21, no. 11, pp. 2546-2556, 2003.

[26] C. Reis, R. P. Dionisio, B. Neto, A. Teixeira, and P. Andre, "All-optical XOR based on integrated MZI-SOA with co- and counter-propagation scheme," in Proceedings of the 3rd ICTON Mediterranean Winter Conference (ICTON-MW '09), 4, p. 1, Angers, France, January 2009.

[27] K. S. Choi, Y. T. Byun, S. Lee, and Y. M. Jhon, "All-optical OR/NOR Bi-functional logic gate by using cross-gain modulation in semiconductor optical amplifiers," Journal of the Korean Physical Society, vol. 56, no. 4, pp. 1093-1096, 2010.

[28] M. J. Connelly, "Wideband semiconductor optical amplifier steady-state numerical model," IEEE Journal of Quantum Electronics, vol. 37, no. 3, pp. 439-447, 2001. 
[29] M. Menif, P. Lemieux, W. Mathlouthi, and L. A. Rusch, "Incoherent-to-coherent wavelength conversion using semiconductor optical amplifier," in Proceedings of the IEEE International Conference on Communications (ICC '04), pp. 1740-1744, June 2004. 

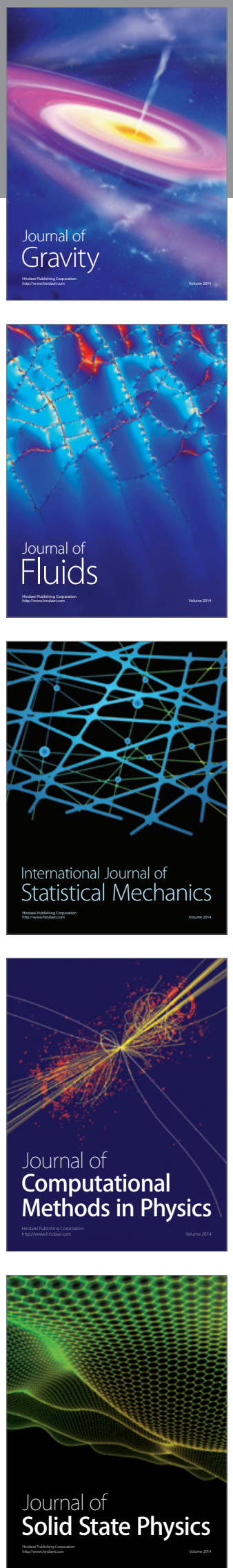

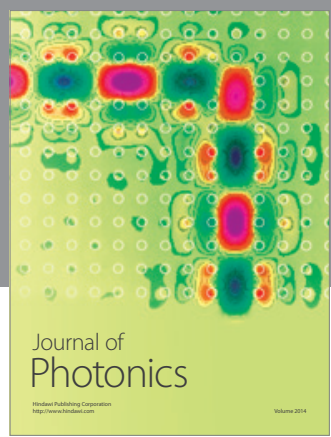

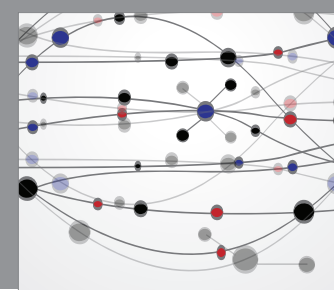

The Scientific World Journal

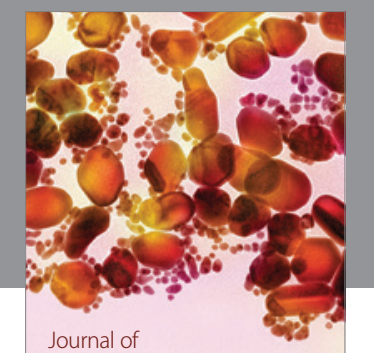

Soft Matter
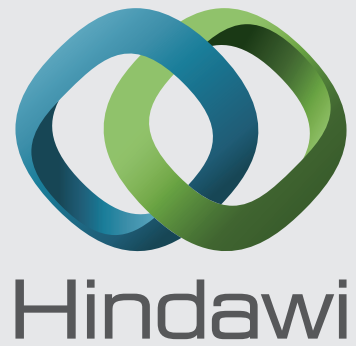

Submit your manuscripts at

http://www.hindawi.com
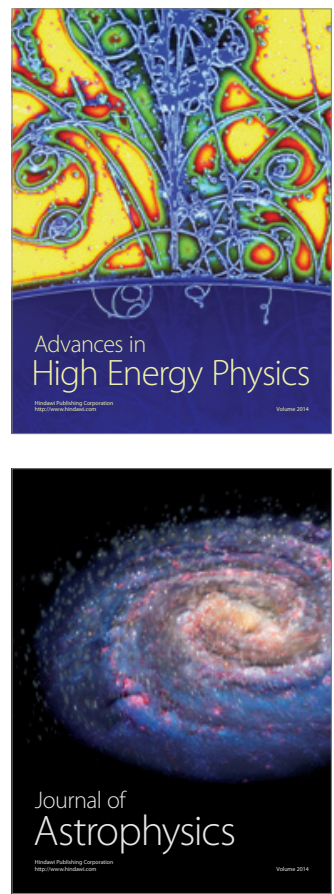
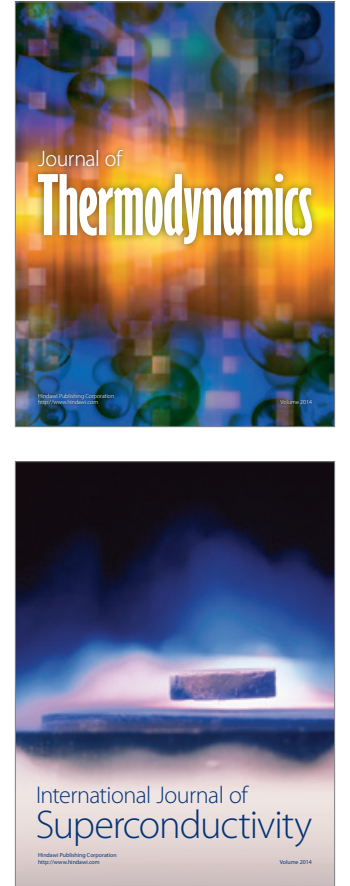
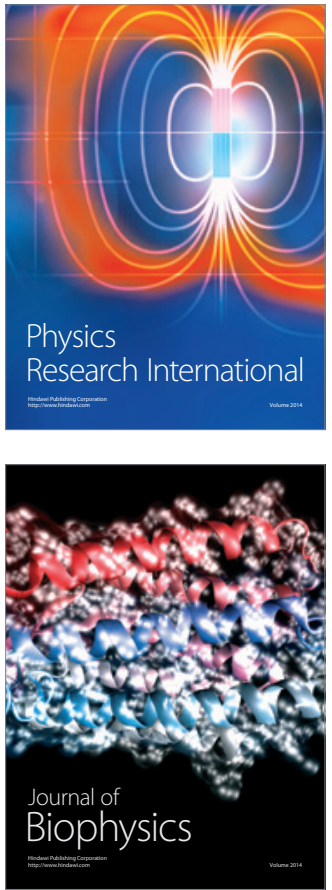
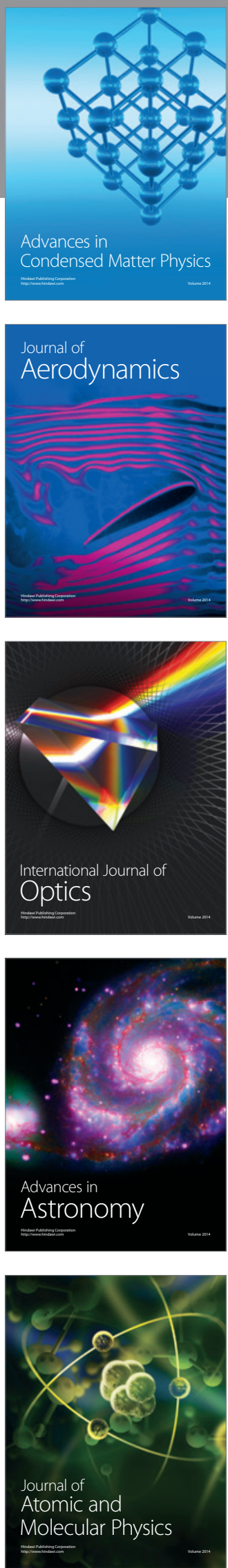